J Cancer Res Clin Oncol (1994) 120:533-538

Cancer Research

Clinical (O)ncology

(C) Springer-Verlag 1994

\title{
The chemosensitizer cyclosporin A enhances the toxic side-effects of doxorubicin in the rat
}

\author{
W. Van de Vrie ${ }^{1,2}$, A.M. Jonker ${ }^{3}$, R.L. Marquet ${ }^{2}$, A.M.M. Eggermont ${ }^{1,2}$ \\ ${ }^{1}$ Department of Surgical Oncology, Rotterdam Cancer Institute, Groene Hilledijk 301, 3075 EA Rotterdam, The Netherlands \\ ${ }^{2}$ Laboratory for Experimental Surgery, Erasmus University, PO Box 1738, 3000 DR Rotterdam, The Netherlands \\ ${ }^{3}$ Department of Pathology, Rotterdam Cancer Institute, Groene Hilledijk 301, 3075 EA Rotterdam, The Netherlands
}

Received: 25 October 1993 / Accepted: 4 February 1994

\begin{abstract}
The feasibility of using chemosensitizers in the circumvention of P-glycoprotein-mediated multidrug resistance has been shown in many studies. We recently reported on the chemosensitizing effect of cyclosporin A (CsA) on doxorubicin in a rat solid tumour model. Using the same experimental design we investigated the side-effects of the combination treatment. During the 35-day experiment doxorubicin treatment caused dose-dependent weight loss, which was enhanced by combination treatment with CsA. The main doxorubicin-related side-effects were myelosuppression (transient leucopenia and thrombopenia) and nephrotoxicity. Damage to the kidney was severe, leading to a nephrotic syndrome and resulting in ascites, pleural effusion, hypercholesterolaemia and hypertriglyceridaemia. These toxicities were enhanced by the addition of the chemosensitizer CsA. Mild doxorubicin-related cardiomyopathy and minimal hepatotoxicity were seen on histological examination. There were no signs of enhanced toxicity of the combination treatment in tissues with known high expression levels of P-glycoprotein, like the liver, adrenal gland and large intestine. CsA had a low toxicity profile, as it only caused a transient rise in bilirubin. In conclusion, the chemosensitizer CsA enhanced the side-effects of the anticancer drug doxorubicin without altering the toxicity pattern. There was no evidence of a therapeutic gain by adding CsA to doxorubicin, compared to single-agent treatment with doxorubicin in $25 \%-33 \%$ higher doses, because of the enhanced toxicity of the combination treatment.
\end{abstract}

Key words: Chemosensitizer - Cyclosporin A - Doxorubicin - Multidrug resistance - Toxicity

\footnotetext{
This work was supported by the Dr Daniël den Hoed Foundation, Rotterdam, The Netherlands

Abbreviations: CsA, cyclosporin A; DOX, doxorubicin; MDR, multidrug resistance; $\mathrm{PBS}$, phosphate-buffered saline

Correspondence to: A.M.M. Eggermont, Department of Surgical Oncology, Rotterdam Cancer Institute, Groene Hilledijk 301, 3075 EA Rotterdam, The Netherlands
}

\section{Introduction}

Multidrug resistance (MDR) is an important mechanism of resistance of tumours to anticancer drugs. In MDR an efflux pump, P-glycoprotein, expels drugs from the cell by active transport (Chin et al. 1993). P-glycoprotein expression has been found in many tumours. High expression levels of Pglycoprotein were demonstrated in colon cancer, renal cell cancer, hepatocellular carcinoma, and adrenocortical cancer, while intermediate levels were found in sarcomas and breast cancer (Fojo et al. 1987; Goldstein et al. 1989). In haematological malignancies, like several leukemias, lymphomas and in multiple myeloma, expression of P-glycoprotein was found in untreated and, to a greater extent, in treated tumours (Dalton et al. 1989; Goldstein et al. 1989; Herweijer et al. 1990). However, this protein is also expressed in normal tissues. Organs with a high expression level of P-glycoprotein are the adrenal gland, liver, kidney, colon and pancreas (Fojo et al. 1987; Thiebaut et al. 1987), and the protein is mainly localized in cells lining excretory lumina, which suggests a detoxification function (Thiebaut et al. 1987).

One way of disturbing the P-glycoprotein-mediated resistance mechanism is by blocking the efflux pump with so-called chemosensitizers. Numerous in vitro studies have shown the efficacy of drugs like verapamil, cyclosporin A (CsA), quinine/quinidine, tamoxifen and others in enhancing the sensitivity of MDR tumour cell lines to anticancer drugs (Ford and Hait 1990). In vivo studies have confirmed the feasibility of reversal of MDR by chemosensitizers in ascites tumour models (Tsuruo et al. 1981; Slater et al. 1986) and in solid tumour models (Osieka et al. 1986; Van de Vrie et al. 1993). In clinical trials promising results have been observed in patients with multiple myeloma, lymphoma and leukemia (Dalton et al. 1989; Solary et al. 1992; Sonneveld et al. 1992; List et al. 1993). In studies with solid tumours chemosensitizers showed less efficacy with responses in a minority of the patients only (Miller et al. 1988; Bissett et al. 1991; Philip et al. 1992; Verweij et al. 1991). Besides, some authors have reported on enhancement of toxic side-effects, like myelosuppression, by the addition of chemosensitizers to the therapeutic regimen (Fiqueredo et al. 1990; Verweij et al. 1991; 
Yahanda et al. 1992). Therefore, the question is raised whether the use of chemosensitizers in combination with anticancer drugs enhances the toxic side-effects of these drugs, apart from enhancing the efficacy of the anticancer treatment. A second question is whether other toxic effects will appear, especially in P-glycoprotein-expressing tissues. Third, chemosensitizers themselves may have adverse effects.

We recently published our results on chemosensitizing in a rat MDR tumour model (Van de Vrie et al. 1993). The chemosensitizer CsA was shown to enhance the cytotoxic efficacy of doxorubicin in vitro and in vivo. A suboptimal dose of doxorubicin $(3 \mathrm{mg} / \mathrm{kg}$ ) was rendered effective against the solid growing CC531 rat colon carcinoma in vivo by the addition of CsA. Drugs were administered intramuscularly and intravenously, which means that, unlike in ascites tumour models, drugs were transported to the tumour and other tissues by a vascular route. Because this is close to the clinical situation, the same model was used to study the toxic effects of the combination treatment on normal tissues in rats. In this study we show that the chemosensitizer CsA enhances the specific toxic effects of doxorubicin on normal tissues, resulting in myelosuppression, severe nephrotoxicity and mild cardiotoxicity. There were no signs of additive toxic damage in tissues with a high expression level of P-glycoprotein, nor of severe CsA-induced toxicity.

\section{Materials and methods}

Animals. Male rats of the inbred WAG/RIJ (RT1 $\left.{ }^{4}\right)$ strain were obtained from Harlan-CPB (Austerlitz, The Netherlands). Animals were bred under specific-pathogen-free conditions and fed standard rat chow (Hope Farms, Woerden, The Netherlands) and water ad libitum. In the experiments rats were $12-18$ weeks old and had a body weight of $220-280 \mathrm{~g}$.

Chemicals. Cyclosporin A was obtained from Sandoz, Basel, Switzerland; doxorubicin (Adriablastina) from Farmitalia, Nivelles, Belgium.

Experimental design. Animals were randomly allocated to the experimental groups. The two control groups consisted of eight animals, while the five experimental groups contained four animals each. The experiment was repeated once. Intravenous injection and blood sampling were done under anaesthetic conditions using ether. Rats were weighed weekly. On days $3,7,14,21$, and 28 a blood sample of $0.75 \mathrm{ml}$ was taken by bleeding from the tail vein. On the 35th day the experiment was terminated and all rats were sacrificed. If an animal was critically ill such that it was not supposed to survive $48 \mathrm{~h}$, or if it had lost approximately $20 \%$ body weight, the animal was sacrificed earlier than day 35 .

Drug treatment. The chemosensitizer CsA, dissolved in olive oil, was injected intramuscularly into the hind leg daily for 3 consecutive days at a dose of $20 \mathrm{mg} / \mathrm{kg}$ body weight. Animals in groups not to be treated with CsA were injected with the vehicle of CsA: olive oil and $6.25 \%$ alcohol. Treatment was given on days $-2,-1$ and 0 . Doxonubicin was administered intravenously on day 0 as a single dose at a concentration of $3 \mathrm{mg} / \mathrm{kg}, 4 \mathrm{mg} / \mathrm{kg}$ or $6 \mathrm{mg} / \mathrm{kg}$ body weight. Control rats were injected with phosphate-buffered saline (PBS). This resulted in the following groups: control (treatment with PBS and vehicle), CsA-con (PBS + CsA), DOX3 (3 mg/kg doxorubicin + vehicle), DOX $3+\mathrm{CsA}(3 \mathrm{mg} / \mathrm{kg}$ doxorubicin + CsA), DOX4 (4mg/kg doxorubicin + vehicle), DOX4+CsA (4 mg/kg doxorubicin + CsA), and DOX6 (6 mg/kg doxorubicin + vehicle).

Haematological and biochemical studies. Blood was collected in lithium/heparin mictotubes (Sarstedt, Germany). The haemoglobin content was determined on the TOA haemoglobin counter HB-100, leucocytes on the Sysmex microcell counter CC-108 and platelets on the TOA platelet counter PL-100 (all Sysmex, TOA Medical Electronics, Hamburg, Germany). The remaining blood sample was centrifuged and serum was collected. Biochemical values of creatinine, urea, aspartate aminotransferase, $\gamma$-glutamyltransferase, total bilirubin, cholesterol and triglyceride were determined on the ELAN-Analyzer (Eppendorf, Hamburg, Germany) with reagents from Merck (Merck Diagnostica, Darmstadt, Germany).

Histology. On day 35 all animals were sacrificed and an autopsy was performed. Ascites and pleural effusion, if present, were aspirated in a syringe and measured. Specimens of the following organs were taken for histological examination: heart, lung, liver, spleen, kidney, large intestine, pancreas and adrenal gland. The organs were removed immediately, fixed in $10 \%$ buffered formalin and embedded in paraffin. Sections were cut at $5 \mu \mathrm{m}$, stained with haematoxylin and eosin, and periodic acid/Schiff. Microscopic sections were coded and scored blindly. The following histological parameters were evaluated: oedema, necrosis, inflammation, accumulation of fat, fibrosis, glycogen storage (liver) and degenerative changes. The extent of damage in kidney and liver was graded semiquantitatively on a 0 to $2+$ scale $(0=$ absent, $1+=$ slightly damaged, $2+=$ severely damaged). The histopathological changes in the heart were assessed according to the scoring system of Bristow et al. (1982), which scale runs from 0 to $3.0+$.

Statistics. Statistical significance was determined with SPSS/PC+, using the Mann-Whitney $U /$ Wilcoxon Rank Sum WTest. $P<0.05$ was considered significant. Results are presented as means with standard deviations. In the line diagrams standard deviations are omitted for readability reasons.

Ethical approval. The experimental protocols adhered to the rules laid down in The Dutch Animal Experimentation Act (1977) and the published Guidelines on the Protection of Experimental Animals by the Council of the EC (1986). Specific protocols were approved by the Committee on Animal Research of the Erasmus University, Rotterdam.

\section{Results}

Weight

Drug treatment had a profound influence on the body weight of the animals. In the first experiment rats treated with the highest doses of doxorubicin and doxorubicin plus CsA did not survive for 35 days (Fig. 1), whereas in the repeat experiment under the same conditions none of the rats died before the end of the experiment. The maximal tolerable dose of doxorubicin in WAG rats is $4 \mathrm{mg} / \mathrm{kg}$. We purposely chose to administer a higher dose for the comparison of toxic side-effects. Rats treated with the highest doses of doxorubicin developed ascites, which made body weight a less reliable parameter towards the end of the experiment for comparison of groups. Therefore, significance of the differences is determined for day 21. All control rats continued to grow during the experiment, while rats treated with doxorubicin lost weight. This effect was most pronounced in the groups DOX6 and DOX4+CsA. The weight curves of the groups DOX $3+$ CsA and DOX 4 ran down in parallel, while the DOX3 group fared better.

\section{Haematological parameters}

Drug treatment had no effect on haemoglobin during the first 14 days (data not shown). The nadir for leucocytes and thrombocytes was reached around day 7 (Table 1). A signifi- 


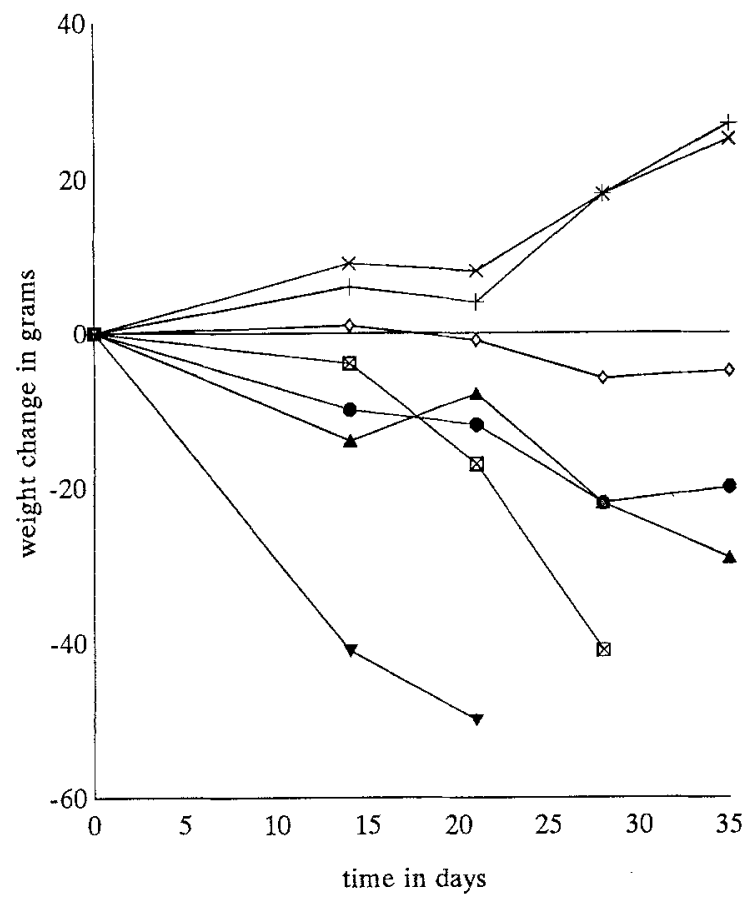

Fig. 1. Body weight changes of rats under treatment with doxorubicin (DOX) with or without cyclosporin A (CsA). + , control; $\times$, CsA-con; $\diamond$, DOX3; $\Delta$, DOX3+CsA; DOX4;, DOX4+CsA; $\bigotimes$, DOX6 (see Materials and methods for definitions of the groups). Statistically significant differences determined on day 21 are: DOX6 versus DOX4+CSA, DOX4, DOX3+CsA, DOX3, CsA-con and control; DOX4+CsA versus DOX4, DOX3+CsA, DOX3, CsA-con, and control; DOX4 versus DOX3, CsA-con, and control; DOX3+CsA versus DOX3, CsA-con and control (all $P<0.025$ )

Table 1. Blood parameters determined on day 7

\begin{tabular}{llll}
\hline Group & Leucocytes & Thrombocytes & Bilirubin \\
\hline Control & $7.76( \pm 0.75)$ & $545( \pm 39)$ & $2.39( \pm 0.28)$ \\
CsA-con & $6.96( \pm 0.66)$ & $497( \pm 33)$ & $3.49( \pm 0.45)^{*^{8}}$ \\
DOX3 & $5.90( \pm 1.80)^{*_{1}}$ & $575( \pm 50)^{*_{1}}$ & $1.60( \pm 0.88)$ \\
DOX3+CsA & $4.68( \pm 1.06)^{*_{2}}$ & $414( \pm 98)^{*_{5}}$ & $2.35( \pm 0.10)$ \\
DOX4 & $5.40( \pm 1.68)^{*_{3}}$ & $415( \pm 74)^{*_{6}}$ & $1.65( \pm 0.17)$ \\
DOX4+CsA & $2.93( \pm 0.25)^{*_{4}}$ & $246( \pm 38)^{*_{4}}$ & $2.08( \pm 0.61)$ \\
DOX6 & $3.20( \pm 0.50)^{*_{4}}$ & $129( \pm 10)^{*_{7}}$ & $1.58( \pm 0.30)$ \\
\hline
\end{tabular}

The groups are defined in Materials and methods

${ }^{*} 1-* 8$ Significance was determined at the $P<0.05$ level: ${ }^{* 1}$ not significantly lower than control groups; ${ }^{{ }^{2}}$ significantly lower than both control groups; ${ }^{* 3}$ significantly lower than control; ${ }^{* 4}$ significantly lower than control groups and DOX3, DOX $3+\mathrm{CsA}$ and DOX $4 ;{ }^{* 5}$ significantly lower than control and DOX $3 ;{ }^{* 6}$ significantly lower than control groups and DOX $3 ;{ }^{* 7}$ significantly lower than all other groups; ${ }^{* 8}$ significantly higher than all other groups

cant drop in leucocyte and platelet count was observed in all drug-treated groups except DOX3. In the groups DOX $3+$ CsA and DOX4 approximately equal levels of leucocytes and platelets were found. The same holds good for leucocytes in the groups DOX4+CsA and DOX6. The DOX4+CsA group had a significant lower nadir compared to the DOX4 group for both haematological parameters.
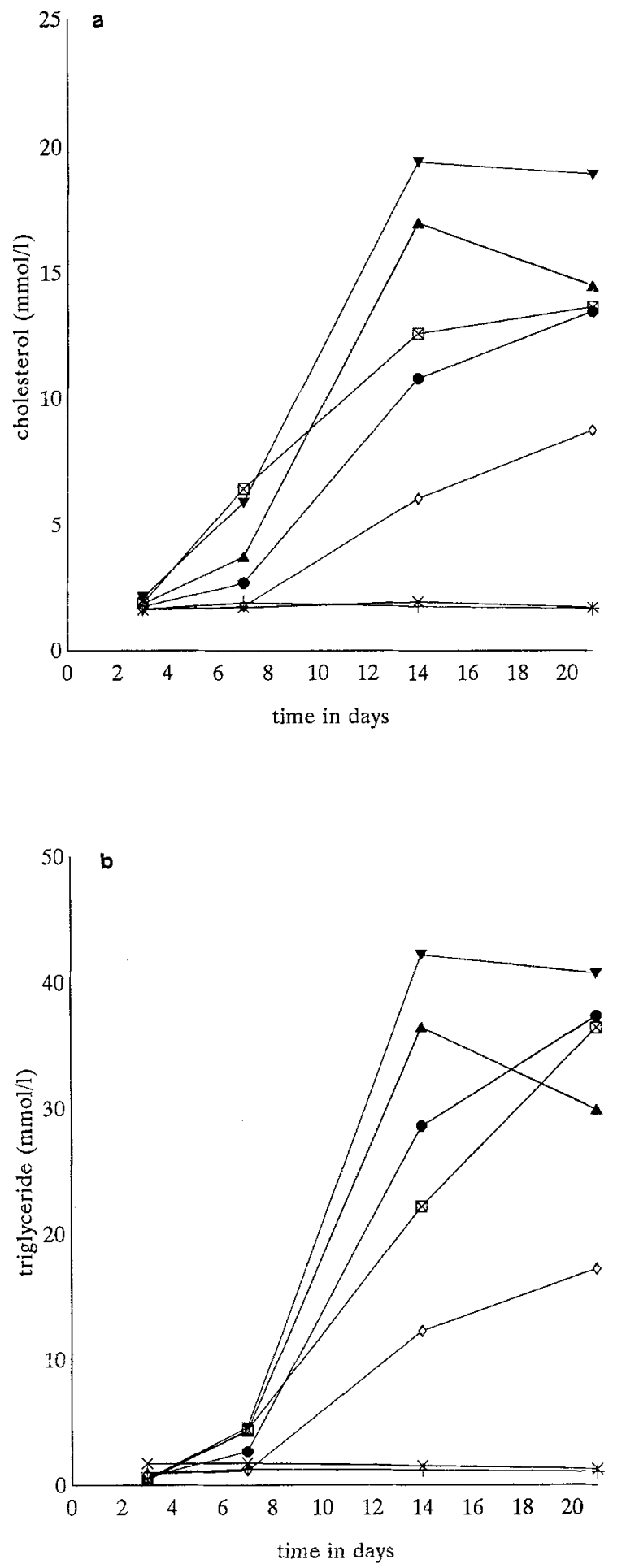

Fig. 2. a Cholesterol concentrations in rats treated with doxorubicin with or without cyclosporin A. Statistical significance between the groups is determined for day 14. All treated groups are significantly different from both control groups $(P<0.01)$; DOX $4+$ CsA versus DOX6, DOX4 and DOX3 $(P<0.025)$; DOX3+CsA versus DOX3 and DOX4 $(P<0.05$; DOX6 versus DOX3 $(P<0.025)$. b Triglyceride concentrations in rats treated with doxorubicin with or without cyclosporin A. Statistical significance between the groups is determined for day 14. All treated groups are significantly different from both control groups $(P<0.01)$; DOX 4+CsA versus DOX6, DOX4 and DOX3 $(P<0.05)$; DOX3+CsA versus DOX $3(P<0.05),+$, Control; $\times$, CsA-con; $\diamond, \operatorname{DOX} 3 ; A$,

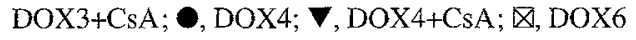




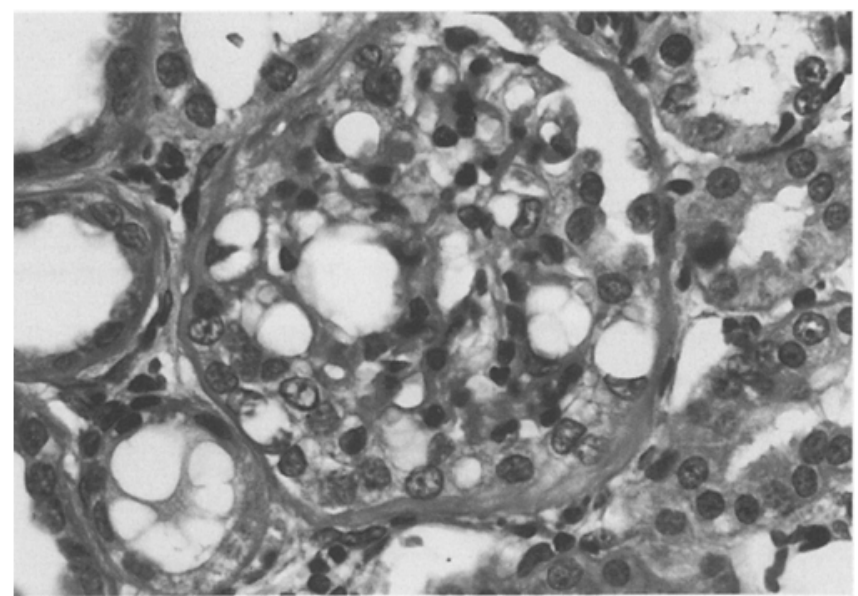

Fig. 3. Nephrotoxicity caused by doxorubicin treatment: thickening of the basement membrane, mesangial hypercellularity, accumulation of lipids and adhesion to Bowman's capsule. Original magnification 400×; $\mathrm{H} \& \mathrm{E}$ staining

\section{Lipid biochemical values}

Doxorubicin had a profound influence on the lipid metabolism in WAG rats. All treated groups had increased levels of serum cholesterol and triglyceride (Fig. 2a,b). The rise continued for 14-21 days and was amplified by CsA: in the DOX4+CsA and DOX3+CsA groups the highest levels were observed. CsA alone had no influence on cholesterol and triglyceride levels.

The high levels of lipids disturbed the measurement of other haematological and biochemical values from 14 days onwards. Therefore, we were only able to determine the short-term effects of the treatment on these values.

\section{Renal biochemical values}

During the first 14 days no significant changes in creatinine and urea were observed (data not shown).

\section{Liver biochemical values}

Doxorubicin treatment had no short-term effect on the liver function parameters aspartate aminotransferase and $\gamma$-glutamyltransferase (data not shown). A significant rise in bilirubin was observed in the CsA control group (Table 1). This cyclosporin-related effect seemed to be mitigated by combination treatment with doxorubicin.

\section{Autopsy}

Pathology data presented here are from the second experiment, in which all animals survived. On day 35 , rats in the groups DOX4+CsA and DOX6 were critically ill. They had lost body weight and subcutaneous fat. During the experiment rats had not had diarrhoea. At autopsy a large amount of ascites and haemorrhagic pleural effusion was found in rats treated with DOX4+CsA and DOX6. Rats in the
DOX4+CsA group had $12.4( \pm 12.4) \mathrm{ml}$ ascites, rats in the DOX6 group $15.3( \pm 6.9) \mathrm{ml}$, while only a small amount of $2.1( \pm 2.4) \mathrm{ml}$ was found in the DOX3+CsA group and no ascites in the other rats. Pleural effusion was $6.5( \pm 3.1) \mathrm{ml}$ and $6.3( \pm 4.4) \mathrm{ml}$ in the DOX4+CsA and DOX6 groups respectively. In the DOX $3+\mathrm{CsA}$ group $0.8( \pm 1.5) \mathrm{ml}$ pleural effusion was found and none in the other rats. The differences in ascites and pleural effusion were statistically significant for the DOX4+CsA and DOX6 groups compared to all other groups except for pleural effusion in DOX6 versus DOX3+CsA. In addition, oedema of the pancreas and paleness of the liver, kidneys and adrenal glands were observed in rats of the DOX4+CsA and DOX6 groups. In the other groups all these macroscopic findings were minimal or absent.

\section{Microscopic study}

Light-microscopic examination of the kidney showed severe damage (2+) in all rats treated with doxorubicin or the combination doxorubicin and CsA, while rats injected with PBS or CsA had normal kidneys. Injured kidneys showed increased glomerular mesangial cellularity, lipid accumulation in macrophages, thickening of basement membranes of glomerular capillaries and Bowman's capsule with in some glomeruli focal adhesions (Fig. 3). The tubule epithelium showed degenerative changes, focal regenerative activity (mitotic figures) and some showed protein casts. In the interstitial space of injured kidneys focal lymphocytic infiltrates were seen. Blood vessels had normal morphology.

The myocardium of rats treated with doxorubicin and the combination doxorubicin plus CsA showed minimal morphological changes with oedema in the interstitial space, slight vacuolisation of myocytes, and sporadic focal inflammation. Necrosis or fibrosis was not observed. The maximal score according to Bristow et al. (1982) was 1.5. Increased doses of doxorubicin revealed the same degree of damage; however, a greater percentage of rats in each group was affected with higher doses (DOX3 25\%; DOX3+CsA 50\%; DOX4 75\%; DOX4+CsA and DOX6 100\%).

Minimal hepatotoxic changes were demonstrated. In rats treated with DOX4 and DOX6 mononuclear inflammation and spotty necrosis were observed with a reduced amount of glycogen (score 2+). Rats treated with the combination doxorubicin and CsA showed slight morphological changes (score 1+) with minor inflammation and sporadic necrotic hepatocytes.

Histological examination of the colon demonstrated oedema in the mucosa of rats treated with doxorubicin and the combination doxorubicin plus CsA, while inflammation or necrosis was absent in this experiment. The lung parenchyma showed some focal inflammatory aggregates not related to the bronchial tree in rats treated with DOX4 and DOX6. The spleen showed slight hypoplasia of the white pulpa in the groups with DOX6 and the combination doxorubicin plus CsA. Pancreas and adrenal glands showed normal histology. The findings in colon, lung and spleen, however, were not observed consistently in all rats within the same treatment group, and differences between the groups were minimal. 


\section{Discussion}

The addition of the chemosensitizer CsA to the anticancer drug doxorubicin clearly enhances its toxicity. In a previous study we demonstrated that the addition of CsA made a suboptimal dose of $3 \mathrm{mg} / \mathrm{kg}$ doxorubicin as effective as $4 \mathrm{mg} / \mathrm{kg}$ doxorubicin (Van de Vrie et al. 1993). In the present study we show data (body weight change curves, haematological parameters, and autopsy findings) indicating that the combination of doxorubicin with CsA is about as toxic as a $25 \%-33 \%$ higher doxorubicin dose alone. We found therefore no therapeutic window, in contrast to Mickisch et al. (1991) in their transgenic mouse model. They had to reduce the dose of most anticancer drugs by $20 \%$, while these doses in combination with D-verapamil reduced MDR cell populations by $44 \%-78 \%$. The results of the combination treatment were favourable compared to results with full doses of the drugs alone. Boesch et al. (1991) reported similar favourable results of a combination treatment of vinblastine and doxorubicin with the CsA analogue PSC 833 in a survival model of mice with MDR tumours.

The toxicity pattern of doxorubicin is not altered by the addition of CsA. Doxorubicin, like most other cytotoxic agents, causes severe damage to cell-renewal systems, which are highly proliferative in postfetal life (Philips et al. 1975). In our experiments the main acute side-effect was myelosuppression with significant leucopenia and thrombopenia. This effect was reversible. No signs of enterocolitis were observed. Unique toxic actions of anthracyclines, especially doxorubicin, are cardiovascular toxic effects, nephrotoxicity and toxic effects on the skeletal system. The last two effects can be observed in several experimental models, while cardiotoxicity is also found in humans (Young 1975). In man cardiomyopathy leading to congestive heart failure is dependent on the total cumulative dose administered (Lenaz and Page 1976). Our study was not designed specifically for studying the toxic effects of doxorubicin on cardiac tissue. The study was short-term, lasting only 35 days, and involved a single-dose treatment schedule for doxorubicin, which made it more apt for studying acute toxic effects than chronic damage. Nevertheless, on microscopic examination mild damage to the cardiac tissue was observed in the most intensely treated rats. Other investigators have found severe cardiomyopathy in rats from 35 days onwards after administering multiple low doses of doxorubicin instead of a single high dose, and reaching higher cumulative values for doxorubicin than we did (Mettler et al. 1977; Van Hoesel et al. 1986). Results in studies with dogs and mice suggest that the addition of the chemosensitizer verapamil potentiates the cardiotoxic effects of doxorubicin (Bright and Buss 1990; Sridhar et al. 1992).

WAG rats proved to be very sensitive to the nephrotoxic effects of doxorubicin, eventually developing a full-blown nephrotic syndrome (Young 1975; Van Hoesel et al. 1986). We were unable to measure proteins and renal parameters after 14 days because of disturbance of the assay caused by turbidity of the hyperlipidaemic serum, but the ascites and pleural effusion indicate hypoproteinaemia. Levels of cholesterol and triglyceride were significantly raised by doxorubicin. Addition of CsA raised the levels of cholesterol and triglyce- ride even higher, in such a way that combination treatment produced the highest levels. Meanwhile, significant amounts of ascites and pleural effusion were only found in the two most intensely treated groups. This indicates that the nephrotoxic effects of doxorubicin were amplified by the chemosensitizer CsA.

Tissues with known high expression levels of P-glycoprotein, like liver, large intestine and adrenal gland, were monitored for toxic effects. No signs of major toxicity were observed. This suggests that these tissues are not susceptible to the cytotoxic effects of doxorubicin, and that raising its intracellular concentration, either by administering a higher dose, or by adding a chemosensitizer, does not make these tissues sensitive to doxorubicin, despite the presence of P-glycoprotein. Other researchers have come to the same conclusion in a pathology study using the chemosensitizer D-verapamil and the drugs vinblastine, doxorubicin, and daunomycin (Mickisch et al. 1991). In contrast, Horton et al. (1989) found enhanced concentrations of vincristine in P-glycoprotein-expressing normal tissues, like small intestine, kidney and liver, caused by the addition of high doses of the chemosensitizer verapamil. Toxicity was enhanced eightfold and symptomatic of vincristine-related neurotoxicity. They did not describe the functional and morphological effects of the raised concentration of intracellular vincristine in these normal tissues. Genne et al. (1989) also reported enhanced doxorubicin accumulation in kidney and liver in combination treatment with the chemosensitizer amiodarone. Combination treatment accelerated doxorubicin-induced death. In clinical studies with chemosensitizers, however, no toxicities, apart from those attributable to the drug or the chemosensitizer, have been observed so far.

CsA seems to produce few toxic effects in the concentrations used for chemosensitizing. In clinical trials steady-state levels from $1000 \mu \mathrm{g} / \mathrm{l}$ up to $5000 \mu \mathrm{g} / \mathrm{l}$ were reported (Verweij et al. 1991; Sonneveld et al. 1992; Yahanda et al. 1992; List et al. 1993). The CsA concentration of $1000 \mu \mathrm{g} / \mathrm{l}$ suffices in vitro for MDR reversal. Side-effects of CsA observed were an early and transient rise in serum bilirubin, without increases in liver enzymes, and hypomagnesaemia (Lum et al. 1992; Sonneveld et al. 1992; Yahanda et al. 1992; List et al. 1993). In our rat study the transient hyperbilirubinaemia appeared to be a purely CsA-dependent feature, which was not enhanced by the addition of doxorubicin. We found no evidence in our rat model for the hypothesis that bilirubin is raised as a consequence of competition between doxorubicin, CsA, and bilirubin at the excretion level, and thus might be used as a marker for P-glycoprotein modulation in vivo (Yahanda et al. 1992; List et al. 1993).

From our studies with doxorubicin and cyclosporin it can be concluded that the addition of a chemosensitizer seriously enhances the toxic side-effects of the anticancer drug without altering the pattern of toxicity. As the toxicity patterns of anticancer drugs are known, side-effects can be anticipated in the planning of clinical trials. However, it remains unclear from this study whether therapeutic gains can be made by the application of a chemosensitizer.

Acknowledgements. We would like to thank W.P. van Schalkwijk and N.M.C. Durante for their skilful technical assistance. 


\section{References}

Bissett D, Kerr DJ, Cassidy J, Meredith P, Traugott U, Kaye SB (1991) Phase I and pharmacokinetic study of D-verapamil and doxorubicin. Br J Cancer 64:1168-1171

Boesch D, Gavériaux C, Jachez B, Pourtier-Manzanedo A, Bollinger P, Loor F (1991) In vivo circumvention of P-glycoprotein-mediated multidrug resistance of tumor cells with SDZ PSC 833. Cancer Res 51:4226-4233

Bright JM, Buss DD (1990) Effects of verapamil on chronic doxorubicin-induced cardiotoxicity in dogs. J Natl Cancer Inst 82:963-964

Bristow MR, Lopez MB, Mason JW, Billingham ME, Winchester MA (1982) Efficacy and cost of cardiac monitoring in patients receiving doxorubicin. Cancer 50:32-41

Chin K-V, Pastan I, Gottesman MM (1993) Function and regulation of the human multidrug resistance gene. Adv Cancer Res 60:157-180

Dalton WS, Grogan TM, Meltzer PS, Scheper RJ, Durie BGM, Taylor CW, Miller TP, Salmon SE (1989) Drug-resistance in multiple myeloma and non-Hodgkin's lymphoma: detection of P-glycoprotein and potential circumvention by addition of verapamil to chemotherapy. J Clin Oncol 7:415-424

Figueredo A, Arnold A, Goodyear M, Findlay B, Neville A, Normandeau R, Jones A (1990) Addition of verapamil and tamoxifen to the initial chemotherapy of small cell lung cancer. A phase I/II study. Cancer 65:1895-1902

Fojo AT, Ueda K, Slamon DJ, Poplack DG, Gottesman MM, Pastan I (1987) Expression of a multidrug-resistance gene in human tumors and tissues. Proc Natl Acad Sci USA 84:265-269

Ford JM, Hait WN (1990) Pharmacology of drugs that alter multidrug resistance in cancer. Pharmacol Rev 42:155-199

Genne P, Coudert B, Pelletier H, Girardot C, Martin F, Chauffert B (1989) Serum concentrations of amiodarone required for an in vivo modulation of anthracycline resistance. Anticancer Res $9: 1655-1660$

Goldstein LJ, Galski H, Fojo A, Willingham M, Lai S-L, Gazdar A, Pirker R, Green A, Crist W, Brodeur GM, Lieber M, Cossman J, Gottesman MM, Pastan I (1989) Expression of a multidrug resistance gene in human cancers. J Natl Cancer Inst 81:116-124

Herweijer H, Sonneveld P, Baas F, Nooter K (1990) Expression of mdr 1 and $\mathrm{mdr} 3$ multidrug-resistance genes in human acute and chronic leukemias and association with stimulation of drug accumulation by cyclosporine. J Natl Cancer Inst 82:1133-1140

Horton JK, Thimmaiah KN, Houghton JA, Horowitz ME, Houghton PJ (1989) Modulation by verapamil of vincristine pharmacokinetics and toxicity in mice bearing human tumor xenografts. Biochem Pharmacol 38:1727-1736

Lenaz L, Page JA (1976) Cardiotoxicity of Adriamycin and related anthracyclines. Cancer Treat Rev 3:111-120

List AF, Spier C, Greer J, Wolff S, Hutter J, Dorr R, Salmon S, Futscher B, Baier M, Dalton W (1993) Phase I/II trial of cyclosporine as a chemotherapy-resistance modifier in acute leukemia. J Clin Oncol 11:1652-1660

Lum BL, Kaubisch S, Yahanda AM, Adler KM, Jew L, Ehsan MN, Brophy NA, Halsey J, Gosland MP, Sikic BI (1992) Alteration of etoposide pharmacokinetics and pharmacodynamics by cyclosporine in a phase I trial to modulate multidrug resistance. J Clin Oncol 10:1635-1642

Mettler FP, Young DM, Ward JM (1977) Adriamycin-induced cardiotoxicity (cardiomyopathy and congestive heart failure) in rats. Cancer Res 37:2705-2713
Mickisch GH, Licht T, Merlino GT, Gottesman MM, Pastan I (1991) Chemotherapy and chemosensitization of transgenic mice which express the human multidrug resistance gene in bone marrow: efficacy, potency, and toxicity. Cancer Res 51:5417-5424

Miller RL, Bukowski RM, Budd GT, Purvis J, Weick JK, Shepard K, Midha KK, Ganapathi R (1988) Clinical modulation of doxorubicin resistance by the calmodulin-inhibitor, trifluoperazine: a phase I/II trial. J Clin Oncol 6:880-888

Osieka R, Seeber S, Pannenbäcker R, Soll D, Glatte P, Schmidt CG (1986) Enhancement of etoposide-induced cytotoxicity by cyclosporin A. Cancer Chemother Pharmacol 18:198-202

Philip PA, Joel S, Monkman SC, Dolega-Ossowski E, Tonkin K, Carmichael J, Idle JR, Harris AL (1992) A phase I study on the reversal of multidrug resistance (MDR) in vivo: nifedipine plus etoposide. $\mathrm{Br} \mathrm{J}$ Cancer 65:267-270

Philips FS, Gilladoga A, Marquardt H, Stemberg SS, Vidal PM (1975) Some observations on the toxicity of Adriamycin (NSC-123127). Cancer Chemother Rep 6:177-181

Slater LM, Sweet P, Stupecky M, Wetzel MW, Gupta S (1986) Cyclosporin A corrects daunombicin resistance in Ehrlich ascites carcinoma. Br J Cancer 54:235-238

Solary E, Caillot D, Chauffert B, Casasnovas R-O, Dumas M, Maynadie M, Guy H (1992) Feasibility of using quinine, a potential multidrug resistance-reversing agent, in combination with mitoxantrone and cytarabine for the treatment of acute leukemia. J Clin Oncol 10:1730-1736

Sonneveld P, Durie BGM, Lokhorst HM, Marie J-P, Solbu G, Suciu S, Zittoun R, Löwenberg B, Nooter K (1992) Modulation of multidrugresistant multiple myeloma by cyclosporin. Lancet 340:255-259

Sridhar R, Dwivedi C, Anderson J, Baker PB, Sharma HM, Desai P, Engineer FN (1992) Effects of verapamil on the acute toxicity of doxorubicin in vivo. J Natl Cancer Inst 84:1653-1660

Thiebaut F, Tsuruo T, Hamada H, Gottesman MM, Pastan I, Willingham MC (1987) Cellular localization of the multidrug-resistance gene product P-glycoprotein in normal human tissues. Proc Natl Acad Sci USA 84:7735-7738

Tsuruo T, Tida H, Tsukagoshi S, Sakurai Y (1981) Overcoming of vincristine resistance in $\mathrm{P} 388$ leukemia in vivo and in vitro through enhanced cytotoxicity of vincristine and vinblastine by verapamil. Cancer Res 41:1967-1972

Van de Vrie W, Gheuens EEO, Durante NMC, De Bruijn EA, Marquet RL, Van Oosterom AT, Eggermont AMM (1993) In vitro and in vivo chemosensitizing effect of cyclosporin $A$ on an intrinsic multidrug-resistant rat colon tumour. J Cancer Res Clin Oncol 119:609-614

Van Hoesel QGCM, Steerenberg PA, Dormans JAMA, De Jong WH, De Wildt DJ, Vos JG (1986) Time-course study on doxorubicin-induced nephropathy and cardiomyopathy in male and female LOU/M/Wsl rats: lack of evidence for a causal relationship. J Natl Cancer Inst 76:299-307

Verweij J, Herweijer H, Oosterom R, Van der Burg MEL, Planting ASTh, Seynaeve C, Stoter G, Nooter K (1991) A phase II study of epidoxorubicin in colorectal cancer and the use of cyclosporin $A$ in an attempt to reverse multidrug resistance. $\mathrm{Br} J$ Cancer 64:361-364

Yahanda AM, Adler KM, Fisher GA, Brophy NA, Halsey J, Hardy RI, Gosland MP, Lum BL, Sikic BI (1992) Phase I trial of etoposide with cyclosporine as a modulator of multidrug resistance. J Clin Oncol 10:1624-1634

Young DM (1975) Pathologic effects of Adriamycin (NSC-123127) in experimental systems. Cancer Chemother Rep 6:159-175 